Archives de sciences sociales des religions

152 | octobre-décembre 2010

Bulletin Bibliographique

\title{
Séverine Mathieu, La transmission du judaïsme dans les couples mixtes
}

Paris, Les Éditions de l'Atelier, 2009, 174 p.

Yves Chevalier

\section{OpenEdition}

\section{Journals}

Édition électronique

URL : http://journals.openedition.org/assr/22070

DOI : $10.4000 /$ assr.22070

ISSN : $1777-5825$

Éditeur

Éditions de l'EHESS

Édition imprimée

Date de publication : 31 décembre 2010

Pagination : 9-242

ISBN : 9782713223013

ISSN : 0335-5985

Référence électronique

Yves Chevalier, "Séverine Mathieu, La transmission du judaïsme dans les couples mixtes », Archives

de sciences sociales des religions [En ligne], 152 | octobre-décembre 2010, document 152-85, mis en

ligne le 10 mai 2011, consulté le 21 septembre 2020. URL : http://journals.openedition.org/assr/22070 ; DOI : https://doi.org/10.4000/assr.22070

Ce document a été généré automatiquement le 21 septembre 2020

(C) Archives de sciences sociales des religions 


\section{Séverine Mathieu, La transmission du judaïsme dans les couples mixtes}

Paris, Les Éditions de l'Atelier, 2009, 174 p.

Yves Chevalier

\section{RÉFÉRENCE}

Séverine Mathieu, La transmission du judaïsme dans les couples mixtes, Paris, Les

Éditions de l'Atelier, 2009, 174 p.

1 Selon des données qui datent cependant de quelques années, on estime que, chez les juifs de France, plus de $40 \%$ des personnes mariées de moins de trente ans ont un conjoint non juif, et ce chiffre serait en augmentation. Ce phénomène, conséquence, entre autres, d'une baisse de la pratique religieuse touchant aussi bien le judaïsme que les autres religions instituées, est lié directement aux transformations de notre société où les identités personnelles ne se construisent plus ou rarement sur les seules appartenances religieuses, mais sur un large éventail de critères disparates. L'individu peut alors, pour lui-même et pour les autres, «se bricoler " une identité composite où les références à une tradition et à une histoire peuvent ne jouer aucun rôle et, dans ce cas, le caractère juif disparaît; ou bien, au contraire, rester opérantes, même si c'est $a$ minima, et la revendication d'un certain «être-juif » persiste alors. D'où, ce qu'avait déjà montré Dominique Schnapper, en 1980, dans Juifs et israélites, la montée d'un sousgroupe, celui des «nouveaux-israélites », pour lequel le judaïsme est une culture et un destin (auxquels, d'ailleurs, le non-juif du couple peut lui aussi adhérer).

2 La question que soulève Séverine Mathieu est de savoir ce que de tels couples peuvent transmettre à leurs enfants: non pas, d'abord, une pratique religieuse - quoique le souvenir de certains rituels puisse être conservé et réaménagé - mais un ensemble de dimensions qui ne sont pas choisies au hasard mais constituent les marqueurs proprement dits de cette judéité en construction. Pour ce faire, elle a entrepris de mener une enquête par entretiens auprès de couples mixtes pour leur demander 
comment ils définissaient eux-mêmes ce qu'ils considéraient comme important et souhaitaient transmettre, d'une manière ou d'une autre, à leurs enfants lorsqu'ils en avaient, s'il y avait, à ce propos, accord ou tension avec le conjoint non juif. Rencontrer des couples mixtes qui acceptent l'entretien n'a pas été facile, nombre de couples contactés refusant en se disant non concernés par le problème. L'auteure a pu, cependant, mener des entretiens approfondis avec vingt-sept couples (plus onze entretiens individuels). Elle insiste pour dire qu'il ne s'agit pas là d'un échantillon représentatif ne serait-ce que du fait de la méthode employée, parce qu'il est exclusivement parisien et essentiellement issu des classes sociales supérieures et des professions intellectuelles. Elle s'est seulement efforcée de diversifier cet échantillon sur les critères de sexe, d'âge et d'origine (entre familles originaires d'Europe et familles issues du bassin méditerranéen). Mais, pour elle, « les acteurs de ce travail sont une bonne illustration de ce qui se joue à l'intérieur d'un couple et d'une famille en matière de transmission, dans un contexte contemporain caractérisé par l'individualisation des pratiques et des identités » (p. 26).

3 L'ouvrage est structuré en cinq chapitres, qui reprennent thématiquement les principaux éléments du discours des personnes interrogées. Un premier chapitre aborde la représentation que le conjoint non juif se fait du judaïsme, et donc de l'identité de l'autre. Ainsi peut émerger ce qui est susceptible de transmission: certaines pratiques culturelles, un prénom; mais aussi ce qui peut être le sujet de controverses et même de conflits (qualifiés cependant de "doux»). Il s'avère ici, visiblement, que la constitution de l'échantillon oriente le contenu des entretiens, puisque, dans l'ensemble, les conjoints non juifs acceptent le judaïsme de l'autre et, même quand il y a désaccord, sont prêts à transmettre le minimum nécessaire à l'élaboration de la judéité. Les quatre chapitres suivants traitent alors des points constitutifs de cette judéité : la circoncision, la cuisine, la Shoah et Israël.

Le rite de la circoncision marque, traditionnellement, l'appartenance au judaïsme; pour la majorité des couples mixtes rencontrés, cette marque d'appartenance n'est pas religieuse mais culturelle - et l'acte lui-même est rationalisé en termes hygiénistes et effectué en milieu hospitalier. Les hommes juifs y sont généralement attachés (même si, la mère étant par définition non juive, l'enfant n'est pas juif selon la Loi), mais aussi les femmes non juives qui, malgré leur réticence pour un acte qu'elles considèrent comme brutal, y voient une manière d'inscrire leur fils dans une histoire et une filiation. Les couples mixtes de l'échantillon qui n'ont pas fait circoncire leur fils restent minoritaires et justifient leur décision autant par le souvenir de la Shoah (la circoncision était à l'époque une stigmatisation) que par la volonté de rupture avec le judaïsme traditionnel. Un second domaine où émerge cette nouvelle façon de s'identifier comme juif est la cuisine. Comme le savent les ethnologues, il s'agit là d'un domaine qui persiste lorsque les autres marqueurs d'appartenance au groupe d'origine ont déjà été effacés. Pour les personnes interrogées par l'auteure, il s'agit, d'une part, de retrouver des sensations de l'enfance et de s'inscrire dans une mémoire familiale, mais aussi, pour le conjoint non juif, de faire l'apprentissage de l'autre, de marquer une appartenance et de s'inscrire dans une mémoire collective. Il ne s'agit pas, à proprement parler, d'un respect de la cacherout - ce qui serait une préoccupation rituelle - mais d'un bricolage autour de la "cuisine juive traditionnelle », mêlée d'ailleurs à d'autres cuisines (en fonction des origines et de l'histoire familiale) et 
autour du calendrier, certaines fêtes (Pessah, Kippour) étant célébrées hors de tout rituel religieux...

5 Les deux autres dimensions de la définition de cette nouvelle judéité renvoient à l'histoire récente du peuple juif : la persécution durant la Seconde Guerre mondiale, qui a largement décimé les familles dont sont issues les personnes interrogées, et la création de l'État d'Israël, qui a, même lorsqu'ils s'en défendent, redessiné pour eux l'identité juive moderne: ils ont "de la famille» là-bas, et ne peuvent rester indifférents à ce qui s'y passe. Dans l'un et l'autre de ces domaines, les attitudes peuvent varier, mais il apparait bien que c'est la fidélité au passé - d'abord familial, ensuite groupal - et au présent qui dicte les choix et les affirmations identitaires, même pour le conjoint non juif qui respecte alors l'être même de leur conjoint juif.

6 En terminant, l'auteur rappelle que, dans un contexte où l'antisémitisme est toujours en action, ses interlocuteurs sont en quelque sorte sommés de prendre position; refusant de se définir traditionnellement, ils se trouvent obligés d'inventer une nouvelle façon d'être juif, pour perpétuer une mémoire et pour s'affirmer porteurs d'une culture qu'ils tendent à transmettre à la génération suivante. Il est indéniable, à partir de l'échantillon interrogé, que ce processus de sécularisation d'une tradition à l'origine religieuse est ici à l'œuvre; la question reste de savoir si ce même processus est présent et fonctionne de la même manière dans les autres sous-groupes non religieux de la population d'origine juive - mais l'enquête, par construction, ne pouvait apporter une réponse. 